\title{
ANÁLISE DA CONVERGÊNCIA DE RENDA NOS MUNICÍPIOS DO RIO GRANDE DO NORTE, BRASIL, ENTRE 1991-2014
}

\section{Diego Palmiere Fernandes}

Universidade Federal da Paraíba - Programa de Pós Graduação em Economia (UFPB/PPGE)

Mossoró - RN - Brasil

E-mail: diego_palmiere@hotmail.com

ORCID: https://orcid.org/0000-0002-7616-5309

\section{Wellington Ribeiro Justo}

Universidade Regional do Cariri (URCA)

Universidade Federal de Pernambuco (UFPE)

Recife - Pernambuco - Brasil

E-mail: justowr@yahoo.com.br

ORCID: https://orcid.org/0000-0002-4182-4466

\section{Roberta de Moraes Rocha}

Universidade Federal de Pernambuco (UFPE)

Recife - Pernambuco - Brasil

E-mail: roberta.mrocha@ufpe.br

ORCID: https://orcid.org/0000-0001-8111-7734

Recebido em: 02/09/2021. Aprovado em: 04/10/2021

DOl: http://dxdoiorg/10.5380/guajuv7i2 81159

\section{Resumo}

O objetivo deste trabalho foi analisar a convergência da renda per capita do Rio Grande do Norte, Brasil, no período de 1991-2014 entre os seus 167 municípios. Metodologicamente, foram utilizados os testes de $\beta$-convergência absoluta, condicional e a hipótese $\sigma$-convergência por Mínimos Quadrados Ordinários (MQO) e técnicas de econometria espacial, aplicando dados do Anuário Estatístico do Instituto de Desenvolvimento Sustentável e Meio Ambiente do Rio Grande do Norte (IDEMA), Instituto Brasileiro de Geografia e Estatística (IBGE) e do Programa das Nações Unidas para o desenvolvimento (PNUD). A hipótese de concentração de renda foi aceita utilizando os três modelos. Os resultados encontrados para os testes de $\beta$-convergência absoluta e condicional indicam que os municípios com menor renda per capita apresentaram taxas de crescimento, ainda que lentas, mais elevadas em comparação com os municípios de maior renda per capita. Esses resultados foram corroborados pelo teste de $\sigma$-convergência, que mostra a 
dispersão da renda no período analisado. Portanto, apesar das desigualdades existentes no estado norte-rio-grandense ainda serem acentuadas, houve nos últimos anos, com base nos resultados obtidos, uma redução deste diferencial.

Palavras-chave: Rio Grande do Norte. Convergência de renda. PIB per capita. Desigualdade regional.

\title{
Analysis of income convergence in municipalities of Rio Grande do Norte, Brazil, between 1991-2014.
}

\begin{abstract}
The objective of this paper was to analyze the convergence of per capita income in Rio Grande do Norte, Brazil, in the period 1991-2014 among its 167 municipalities. Methodologically, the tests of absolute $\beta$-convergence, conditional and the hypothesis $\sigma$-convergence by Ordinary Least Squares (OLS) and spatial econometric techniques, using data from the Statistical Yearbook of the Institute for Sustainable Development and Environment of Rio Grande do North (IDEMA), Brazilian Institute of Geography and Statistics (IBGE) and the United Nations Development Program (UNDP). The hypothesis of income concentration was accepted using the three models. The results found for the absolute and conditional $\beta$-convergence tests indicate that municipalities with lower per capita income presented higher, albeit slow, growth rates compared to municipalities with higher per capita income. These results were corroborated by the $\sigma$-convergence test, which shows the income dispersion of per capita in the analyzed period. Therefore, despite the existing inequalities in the state of Rio Grande do Norte are still accentuated, there has been in recent years, based on the results obtained, a reduction in this differential.
\end{abstract}

Keywords: Rio Grande do Norte. Income convergence. GDP percapita. Regional inequality. 


\section{Introdução}

Os estudos sobre disparidades econômicas e convergência de renda entre países e regiões têm ocupado grande espaço na literatura econômica desde os anos 1980 . A questão-chave que se coloca nesses estudos é a de saber se as economias têm tendência a convergir em direção aos mesmos níveis de renda (ou produto per capita ${ }^{1}$ ), ou seja, se existe algum mecanismo que permita que as economias menos desenvolvidas alcancem o nível de produto das economias mais desenvolvidas (STÜLP E FOCHEZATTO, 2004).

No debate de convergência, surgem duas definições principais: a convergência absoluta e a convergência condicional. A primeira ocorre quando o nível de renda per capita de um país pobre alcança o de um país rico, que pode ser verificado se a taxa de crescimento do país em desenvolvimento é significativamente maior que a do país desenvolvido. Por outro lado, a segunda implica que cada país está convergindo para o seu próprio estado estacionário e que no longo prazo todas as taxas de crescimento serão equalizadas (CASAGRANDE; HOECKEL; SANTOS, 2013).

Essas diferentes versões de convergência são fundamentadas nos modelos de crescimento neoclássico do tipo Solow (1956) e Swan (1956), que mostram que as taxas de crescimento do produto de diferentes países ou regiões tendem a se igualar no equilíbrio de longo prazo. Nesses modelos, a centralização da renda per capita ocorreria por causa da hipótese de retornos decrescentes dos fatores produtivos (capital físico e trabalho). Segundo Santos et al. (2012), uma vez que as regiões menos desenvolvidas utilizam esses fatores menos intensivamente, elas tenderiam a crescer mais rapidamente que as regiões ricas, de modo a alcançar o nível de renda per capita destas no longo prazo.

Conforme Matos Filho et. al. (2012), para o Brasil, a análise do crescimento econômico mostra-se importante para regiões, estados e/ou municípios, uma vez que o aumento da renda per capita está quase sempre correlacionado com uma melhoria dos padrões de vida. Nesse contexto, a confirmação ou a negação de um processo de concentração do produto é importante para a formulação e implementação de políticas públicas que visem a proporcionar a redução das disparidades de renda existentes dentro do país ou de uma mesma região.

A desigualdade de renda é um problema de toda a sociedade brasileira e não poderia ser diferente no Rio Grande do Norte - RN. O estado é composto por 167 municípios

1 Indicador utilizado para medir o grau de desenvolvimento de um país, obtido a partir da divisão da renda total pela população (SANDRONI, 2005). 
e possui uma população estimada de 3.474 .998 milhões de habitantes distribuídos em uma área de $52.811,110 \mathrm{~km}^{2}$ (IBGE, 2016).

Segundo os dados do Produto Interno Bruto (PIB) de 2014, apresentados pelo IBGE, em parceria com o Instituto de Desenvolvimento Sustentável e Meio Ambiente (Idema), o PIB do RN alcançou $\mathrm{R} \$ 54,02$ bilhões, representando 0,9\% do PIB brasileiro. Em termos reais, houve um aumento de 1,6\%, sendo que o valor adicionado bruto e os impostos (líquidos de subsídios) sobre produto cresceram 1,1\% e 5,4\%, respectivamente. Já o PIB per capita de 2014 foi de $\mathrm{R} \$ 15.849,33$. Ademais, seu produto apresentou uma variação real anual de $(1,6 \%)$ maior que a taxa do Brasil (0,5\%) e inferior à da Região Nordeste (2,8\%) em comparação com o ano anterior, conforme pode ser visto na Tabela 1.

TABELA 1 - PIB A PREÇOS DE MERCADO BR, NE E RN (2013-2014)

\begin{tabular}{|c|c|c|c|c|c|c|}
\hline \multirow[b]{2}{*}{$\begin{array}{l}\text { Brasil, } \\
\text { Regiões } \\
\text { do } \\
\text { Nordeste }\end{array}$} & \multicolumn{4}{|c|}{ Produto Interno Bruto } & \multirow[b]{2}{*}{$\begin{array}{c}\text { População } \\
\text { residente } \\
\text { (1.000hab.) } \\
2014\end{array}$} & \multirow[b]{2}{*}{$\begin{array}{l}\text { Produto } \\
\text { Interno } \\
\text { Bruto per } \\
\text { capita } \\
2014 \text { R\$ }\end{array}$} \\
\hline & $\begin{array}{c}\text { Preços } \\
\text { correntes } \\
2014 \\
(1.000,00 R \$)\end{array}$ & $\begin{array}{c}\text { Variação real } \\
\text { anual } \\
2014 / 2013 \\
\text { (\%) }\end{array}$ & $\begin{array}{l}\text { Variação real } \\
\text { acumulada } \\
\text { 2002-2014 } \\
(\%)\end{array}$ & $\begin{array}{c}\text { Variação real } \\
\text { média ao ano } \\
2002-2014 \\
(\%)\end{array}$ & & \\
\hline Brasil & $5.778,953$ & 0,5 & 50,7 & 3,5 & 202.769 & $28.500,24$ \\
\hline Nordeste & 805.099 & 2,8 & 58,8 & 3,9 & 56.186 & $14.329,13$ \\
\hline MA & 76.842 & 3,9 & 84,0 & 5,2 & 6.851 & $11.216,37$ \\
\hline $\mathrm{PI}$ & 37.723 & 5,3 & 86,4 & 5,3 & 3.195 & $11.808,08$ \\
\hline CE & 126.054 & 4,2 & 62,6 & 4,1 & 8.843 & $14.255,05$ \\
\hline $\mathrm{RN}$ & 54.023 & 1,6 & 43,2 & 3,0 & 3.409 & $15.849,33$ \\
\hline PB & 52.936 & 2,9 & 72,5 & 4,6 & 3.944 & $13.422,42$ \\
\hline PE & 155.143 & 1,9 & 53,4 & 3,6 & 9.278 & $16.722,05$ \\
\hline$A L$ & 40.975 & 4,8 & 49,9 & 3,4 & 3.322 & $12.335,44$ \\
\hline SE & 37.472 & 0,4 & 54,2 & 3,7 & 2.220 & $16.882,71$ \\
\hline BA & 223.930 & 2,3 & 52,6 & 3,6 & 15.126 & $14.803,95$ \\
\hline
\end{tabular}

FONTE: IDEMA (2014).

De acordo com os dados do IDEMA (2014), o RN tem uma economia composta predominantemente por serviços e atividades industriais, que correspondem a cerca de 74,5\% do PIB estadual. Analisando o PIB dos municípios foi possível perceber como de fato é grande a diferença do nível de renda entre os municípios norte-rio-grandenses, dos 167 municípios do RN em somente três está concentrado 55\% do PIB, que são a Capital Natal, Parnamirim e Mossoró. 
Diante deste cenário, pode-se constatar evidências de trajetórias de crescimento diferentes dentro do estado, apontando que o crescimento econômico não ocorre de maneira equilibrada em todas as regiões. Isso pode ser o reflexo de diferentes estruturas produtivas a nível regional, em que as mudanças setoriais influenciam a configuração espacial da produção e da renda.

Nesse contexto, o objetivo geral deste estudo é verificar a existência de um processo de confluência entre as rendas per capita dos 167 municípios norte-rio-grandenses por meio da taxa de crescimento do produto entre os anos 1991 e 2014, através do arcabouço teórico da $\beta$-convergência absoluta, condicional e da $\sigma$-convergência, considerando a perspectiva espacial na análise, bem como, especificamente, analisar se há presença de concentração de renda municipal em uma modelagem, considerando a dependência espacial dos dados; identificar possíveis fatores que sejam responsáveis pelo (de)crescimento municipal e desigualdade entre eles; e na hipótese de fraca concentração, identificar a ocorrência de polarização dos municípios.

No âmbito desse estudo, faremos o uso de ferramentas de econometria espacial que têm sido utilizadas na análise da hipótese de convergência de renda, dado que a abordagem tradicional não tem considerado explicitamente o espaço em que as relações ocorrem. Diversos estudos, cabe destacar Rey e Montouri (1999), Clinch e O’Neill (2009), Ertur e Koch (2007) e Lim (2016), têm aplicado técnicas econométricas espaciais para explorar o processo dinâmico de afluência de renda regional, oferecendo testes de robustez que melhoram a compreensão deste processo de centralização da riqueza.

Essa é a primeira iniciativa de estudos referentes à convergência de renda para o conjunto dos municípios norte-rio-grandenses. Dessa forma, questiona-se se o crescimento econômico do estado, observado nos últimos anos, tem favorecido para a redução das discrepâncias de renda entre os municípios.

Pretende-se colaborar com a literatura nacional sobre convergência de renda, e para a economia regional, ao adotar técnicas de econometria espacial e auxiliar na análise da concentração ou não da renda municipal.

O trabalho está dividido em sete seções, além dessa seção introdutória, a segunda seção apresenta uma revisão da literatura; a seção três mostra o método utilizado; a seção quatro apresenta os dados utilizados na pesquisa; a seção cinco discute os principais resultados; a seção seis as conclusões; e, finalmente, a seção sete apresenta as referências bibliográficas utilizadas. 


\section{Revisão da literatura}

O tema "convergência" é amplamente abordado, tanto na literatura internacional como nacional. As origens dos modelos de crescimento e convergência são fundamentadas nos modelos neoclássicos de crescimento de Solow (1956), os quais mostram que as taxas de crescimento da renda ou produto per capita de diferentes regiões geográficas tendem a se igualar no equilíbrio de longo prazo (estado estacionário). Segundo o autor, isso ocorre porque há retornos decrescentes dos fatores produtivos, e é por isso que uma região menos desenvolvida tende a crescer mais rapidamente que outra mais rica (CASAGRANDE; HOECKEL; SANTOS, 2013).

O estudo sobre convergência permite verificar se uma dada variável como a renda, apresentando diferentes valores para as diferentes regiões ou grupos, teria com o tempo uma diminuição nessa diferença, indicando que a desigualdade diminui. Portanto, permite analisar a dinâmica da desigualdade entre diferentes grupos ou regiões (LOPES, 2004).

Como ponto de partida para a literatura internacional, a análise de $\beta$-convergência absoluta ganhou destaque após o trabalho seminal de Baumol (1986), em que o autor analisou o processo de concentração em 16 países industrializados, entre 1870 e 1979. Posteriormente ao trabalho de Baumol (1986), diversas críticas surgiram à teoria de $\beta$-convergência absoluta, alegando que, por esta ser baseada em regressões de corte transversal ${ }^{2}$ (isto é, características distintas), estaria assumindo que o coeficiente estimado é igual para todas as economias.

Dos críticos ao modelo de $\beta$-convergência absoluta, Barro e Sala-i-Martin (1992) apontam que é mais provável que este modelo se aplique a todas as regiões dentro dos países do que entre os países. Os autores examinaram a concentração de renda per capita para 48 estados americanos no período de 1840 a 1963. Outro estudo dos mesmos autores, detectou a existência de convergência absoluta entre os estados americanos no período de 1960 a 1985. Ainda, Sala-i-Martin (1996) obteve os mesmos resultados para um grupo de países da Organização para a Cooperação e Desenvolvimento Econômico (OCDE) para o período de 1960 a 1990 (CASAGRANDE; HOECKEL; SANTOS, 2013).

2 Dados de corte transversal ou cross-section são uma amostra de dados usados em estatística ou econometria, sejam indivíduos, consumidores, empresas, cidades, etc., em um dado ponto no tempo. Dados em um determinado ponto do tempo são importantes para testar hipóteses e avaliar políticas (WOOLDRIGDE, 2010). 
Além do conceito de $\beta$-convergência, outro termo bastante utilizado na literatura éa $\sigma$-convergência ${ }^{3}$. Esta última ocorre quando a dispersão da renda (ou qualquer outra análise de interesse) entre um grupo de economias se reduz ao longo do tempo, sendo calculada pelo desvio padrão da renda per capita na data inicial e final do período considerado; enquanto a $\beta$-convergência ocorre quando a taxa de crescimento da renda e o nível de renda inicial é negativo.

As abordagens empíricas sobre essas hipóteses também estão presentes no âmbito da economia brasileira, aparecendo em maior número após a década de 90 . Em geral, os trabalhos têm focado principalmente no comportamento da renda per capita entre os estados brasileiros, devido à dificuldade de obtenção de dados em um nível menos desagregado. Dentre eles, podem ser citados Ferreira e Ellery Jr. (1996), Azzoni et al. (2000), Ferreira (2000), Nunes e Nunes (2004) e Santos e Carvalho (2007), que identificaram um processo de $\beta$-convergência absoluta.

Já em relação aos estudos que observaram convergência condicional, sugerindo que os estados brasileiros estariam convergindo para o seu próprio nível de renda em estado estacionário, destacam-se os trabalhos de Abitante (2007), Barreto e Almeida (2008), Cravo e Soukiazis (2006), Resende (2011) e Silveira Neto e Azzoni (2011).

No que se refere a análise de convergência acerca dos municípios brasileiros em sua totalidade, cabe citar Chagas e Toneto Júnior (2003), que constataram a existência de convergência para os municípios brasileiros no período 1980-1991, com as áreas de menor renda crescendo a taxas superiores às de renda inicial maior. Outro trabalho que alcançou destaque no âmbito municipal foi Coelho e Figueiredo (2007), que identificaram a formação de clubes de convergência entre 1970 e 2000. No trabalho, o clube de baixa renda foi formado predominantemente pelos municípios das Regiões Norte e Nordeste, enquanto o outro, com nível de renda maior, foi formado basicamente pelos Municípios das Regiões Centro-Oeste, Sudeste e Sul.

Outros trabalhos a nível nacional têm sido desenvolvidos a fim de se obter evidências particulares e locais. Perobelli, Ferreira e Faria (2007), recorrendo ao método da análise exploratória de dados espaciais, investigaram o comportamento do PIB per capita entre os municípios mineiros, tendo como objeto de análise o período 1975-2003. Os resultados não indicaram sinais de convergência, demonstrando um aumento nas disparidades regionais do estado nesse período.

3 Segundo Barro e Sala-i-Martin (1995), a convergência será calculada pelo desvio padrão da renda per capita na data inicial e final do período considerado; ocorre convergência quando o desvio padrão no período final diminui. 
De Oliveira, Meyer e Paixão (2019), em seu trabalho buscaram trazer evidências da existência de centralização de renda para os municípios do estado do Paraná, tanto em nível estadual como pela divisão em mesorregiões, no período de 2002 a 2015. Seus resultados indicaram que houve $\beta$-convergência absoluta e condicional, com os municípios mais pobres apresentando taxas de crescimento superiores aos municípios mais desenvolvidos. Esses resultados foram fortalecidos pelo teste de $\sigma$-convergência, pois a dispersão da renda per capita reduziu-se significativamente no período analisado.

Trabalho com destaque para a região Nordeste podemos citar, dentre outros, Reis, Araújo e Lima (2018), que analisaram se o crescimento da região Nordeste ocorreu de uma maneira a diminuir as disparidades de rendas entre os municípios no período de 2010-2015. Os resultados mostraram que, para a região como toda, os municípios apresentam os dois tipos de $\beta$-convergência, além do $\sigma$-convergência.

Filho, Silva e Carvalho (2012) analisaram a convergência de renda per capita entre as microrregiões da Região Nordeste do Brasil no período de 1985 a 2008. Para isso foram executadas regressões em cross section entre a taxa de crescimento do PIB entre 1985 e 2000 e o seu nível em 1985, para três tipos de amostras. Em todas as regressões foram constatados processos de -convergência, o que foi confirmado posteriormente por testes de -convergência.

Gomes (2013) analisa a hipótese da convergência do PIB per capita nos estados nordestinos, no período de 1995-2009, com foco no capital humano. Os resultados apontaram para a existência de convergência- $\sigma, \beta$-absoluta e $\beta$-condicional, obtendo, no caso da convergência absoluta, taxas de crescimento bem superiores às encontradas na literatura empírica.

Cruz (2013) analisa o processo de convergência da renda proveniente do trabalho nos municípios nordestinos entre os anos 2000 e 2010, considerando-se os efeitos das interações espaciais existentes entre os municípios desta região, utilizando as técnicas de Análise Exploratória de Dados Espaciais (AEDE) e os modelos desenvolvidos na Econometria Espacial, além de Regressões Ponderadas Geograficamente (RPG). Os resultados encontrados indicam a presença de um processo de convergência muito lento entre os municípios nordestinos.

Assim, constata-se que nos diversos trabalhos de convergência de renda entre os estados e municípios brasileiros existem conclusões coincidentes, que esse processo de centralização é fraco devido à velocidade de concentração, ou seja, a redução da desigualdade é lenta ao longo dos anos. No âmbito municipal, pelo inexpressivo número 
de trabalhos publicados, alguns deles apontam para a divergência e a polarização de convergência (clubes de convergência), e isso se deve à persistente desigualdade regional econômica existente nas regiões brasileiras.

\section{Metodologia}

Recentemente, ferramentas de econometria espacial têm sido utilizadas na análise da hipótese de convergência, dado que a abordagem tradicional de análise da hipótese de convergência de renda não tem considerado explicitamente o espaço em que as relações ocorrem. Diversos estudos, cabe destacar Rey e Montouri (1999), Clinch e O'Neill (2009), Ertur e Koch (2007) e Lim (2016), têm aplicado técnicas econométricas espaciais para explorar o processo dinâmico de concentração de renda regional.

O default da abordagem empírica consiste em testar, inicialmente, a hipótese da $\beta$-convergência absoluta, através da regressão das taxas de crescimento das economias em análise sobre seus respectivos níveis iniciais de renda. Por outro lado, a hipótese da $\beta$-condicional implica que as economias com níveis de renda mais distantes de seus estados estacionários terão taxas de crescimento maiores que aquelas localizadas mais próximas a eles, ou seja, são incluídas variáveis de controle para representar o estado estacionário de cada economia (COELHO; FIGUEREDO, 2007).

\subsection{Estratégia empírica}

Para se analisar o processo de concentração de renda, neste estudo serão utilizados basicamente os testes de ampla abordagem na literatura econômica ( $\beta$-convergência absoluta e condicional e da $\sigma$-convergência). Assim, esta análise se restringirá, em um primeiro momento, ao método sintetizado por Sala-i-Martin (1996), em que são introduzidos controles que condicionam a convergência de renda, tanto associados a capital humano como a capital físico. E, num segundo momento, a estimação é controlada pela autocorrelação espacial (CASAGRANDE; HOECKEL; SANTOS, 2013).

\subsection{Convergência absoluta e condicional padrão}

A definição de $\beta$-convergência absoluta implica na existência de uma correlação negativa entre o nível da renda no início de um período e a taxa de crescimento da renda 
nesse mesmo período, ou seja, países ou regiões com rendas mais baixas crescem a taxas mais elevadas que os ricos. O teste para a verificação da $\beta$-convergência de renda entre os municípios do Rio Grande do Norte seguirá, em um primeiro momento, Barro e Sala-iMartin (1992), numa análise de dados cross-section, apresentando o nível inicial de renda por habitante como principal variável explicativa.

A variável dependente será a taxa de crescimento per capita das rendas municipais, para o período de 1991 a 2014. Inicialmente, a hipótese de $\beta$-convergência é testada de forma tradicional por meio de um modelo de regressão linear simples pelo qual estima-se a taxa de crescimento da renda per capita em relação à renda per capita inicial da região, pelo método de Mínimos Quadrados Ordinários (MQO), da mesma forma que em Barro e Sala-iMartin (1992). A equação básica deste teste é expressa por:

$$
\frac{1}{T} \ln \left(\frac{y_{i, t+1}}{y_{i, 0}}\right)=\beta_{1}+\beta_{2} \ln \ln \left(Y_{i, 0}\right)+\mu_{i}
$$

Em que:

$y_{i, t+1}=$ Renda per capita no período inicial;

$y_{i, 0}=$ Renda per capita no período final;

$T$ = Número de anos entre o período inicial e final da observação amostral;

$\mu_{i}=$ Erro aleatório.

O lado esquerdo da equação (1) corresponde à taxa de crescimento da renda per capita. Uma correlação negativa entre a taxa de crescimento e a renda per capita inicial $\left(\beta_{2}<0\right)$ indica que está ocorrendo $\beta$-convergência absoluta (BARRO; SALA-I-MARTIN, 1992).

Segundo esse modelo, existe $\beta$-convergência se $\beta_{2}$ é negativo e estatisticamente significativo, já que, nesse caso, a taxa média de crescimento da renda per capita entre o período inicial e o período final é negativamente correlacionada com seu o nível inicial.

A heterogeneidade nas características populacionais e econômicas entre os municípios norte-rio-grandenses pode implicar que as suposições subjacentes às estimativas descritas pela $\beta$-convergência absoluta podem não ser de tal magnitude. Se os municípios não compartilham a mesma renda per capita de estado estacionário, as estimativas podem sofrer do viés de variável omitida e, em particular, podem levar à rejeição incorreta da hipótese de convergência (SALA-I-MARTIN, 1996). 
Assim, estende-se a análise de convergência entre os municípios do RN controlando por diferenças no nível do produto de estado estacionário de cada município. Em particular, para a análise da $\beta$-convergência condicional, conduz-se a estimação da seguinte equação:

$$
\frac{1}{T} \ln \left(\frac{y_{i, t+0}}{y_{i, 0}}\right)=\beta_{1}+\beta_{2} \ln \ln \left(Y_{i, 0}\right)+\sum_{i=1}^{k} \beta_{k} X_{i, t}+\mu_{i}
$$

$$
\begin{aligned}
& \text { Em que: } \\
Y_{i, 0}= & \text { Renda per capita no período inicial; } \\
y_{i, t+0}= & \text { Renda per capita no período final; } \\
T= & \text { Número de anos entre o período inicial e final da observação amostral; } \\
X_{i, t}= & \text { Grupo de variáveis de controle; } \\
\mu_{i}= & \text { Erro aleatório. }
\end{aligned}
$$

A estimação da $\beta$-convergência condicional inclui como variáveis independentes tanto o nível inicial de renda per capita, quanto um conjunto de variáveis. Este grupo de variáveis inclui: (e_anosestudo) $=$ Anos de Estudos em 1991; $($ Espvida $)=$ Esperança de Vida ao Nascer. Deste modo, tendo estimado o valor da estatística $\beta_{2^{\prime}}$ é possível determinar a velocidade de convergência ( $\theta$ ) (CASAGRANDE; HOECKEL; SANTOS, 2016).

A inclusão dessas variáveis está na importância de tratar o número de anos de estudo concluídos como sendo a razão entre a soma do número de anos de estudo para a população de 25 anos ou mais de idade e o total das pessoas nesse segmento etário, e da esperança de vida ao nascer que corresponde ao número médio de anos que as pessoas viveriam a partir do nascimento (MIRANDA; MAGALHÃES, 2009).

Conforme Miranda e Magalhães (2009) a esperança de vida é uma variável econômica crucial ao desenvolvimento econômico, pois influencia a decisão dos indivíduos quanto ao tempo que permanecerão estudando. Para Mincer (1974), os indivíduos decidem quantos anos estudarão ao comparar seu custo de oportunidade (os salários perdidos mais as taxas escolares) com seu benefício (o aumento no valor presente dos fluxos salariais que receberão até o fim de suas vidas). Um aumento na Expectativa de Vida, tudo o mais constante, eleva o valor presente dos fluxos salariais e, assim, o acúmulo de capital humano.

Deste modo, tendo estimado o valor da estatística $\beta_{2^{\prime}}$ é possível determinar a velocidade de convergência $\theta$ definida por: 


$$
\theta=-\frac{\ln \left(1+T \beta_{2}\right)}{(T)}
$$

Em que:

$\theta \quad=$ Velocidade de convergência de renda;

$\beta_{2}=$ é o coeficiente estimado para o logaritmo natural do PIB per capita inicial, estimado pelas equações (1) e (2);

$T=$ Números de anos.

De acordo com Casagrande et al. (2013), ainda é possível calcular o tempo necessário para que as economias percorram metade do caminho que as separam de seus estados estacionários. Esse valor é denominado de meia-vida, e é determinado por:

$$
\tau=-\frac{\ln \ln (2)}{\left(1+\beta_{2}\right)}
$$

O teste de $\beta$-convergência absoluta dado pela expressão (4) considera que todas as unidades geográficas analisadas possuem o mesmo nível de renda per capita em estado estacionário e que as diferenças observadas nos níveis de produto atuais se devem apenas a desvios de curto prazo no estoque de capital físico das regiões em relação a seus níveis em estado estacionário (CASAGRANDE; HOECKEL; SANTOS, 2016).

\section{$3.3 \beta$ convergência: abordagem espacial}

Diferentemente da econometria tradicional, a econometria espacial concentra-se na preocupação de se incorporar na modelagem o padrão de interação socioeconômica entre os agentes, denominada de autocorrelação espacial, assim como as suas características no espaço, denominado de heterogeneidade espacial. Portanto, estas interações e as características estruturais geram efeitos espaciais que influenciam vários processos econômicos (ANSELIN; BERA, 1998).

Desse modo, seguindo Casagrande et al. (2013), o presente estudo aborda a análise da convergência de renda, absoluta e condicional, controlando-se os efeitos espaciais, seguindo Rey e Montouri (1999). Portanto, nosso ponto de partida é a estimação da equação (1) e (2), nas quais são aplicados testes de diagnósticos de dependência espacial, a saber: 
o teste I de Moran ${ }^{4}$, aplicado aos resíduos da estimação Ordinary Least Squares (OLS); o teste do Multiplicador de Lagrange robusto (LM) para a variável dependente; e, o teste do Multiplicador de Lagrange robusto (LM) para o termo de erro (KOPCZEWSKA, 2021).

Os modelos com dependência espacial são estimados via Máxima Verossimilhança (MV). A literatura de econometria espacial apresenta, basicamente, três especificações distintas de regressão espacial. Tais especificações, segundo Anselin (1998), englobam o modelo de erro espacial (incorporação de uma variável para capturar os efeitos de vizinhança nos erros, dado pela equação 5), modelo lag espacial (efeitos vizinhança na variável dependente, equação 6), o modelo SAR-GMM (estimado pelo método dos momentos generalizados ${ }^{5}$ ) e a equação (7) consistindo o modelo SARAR-ML (que incorpora tanto a defasagem da variável dependente como a autocorrelação espacial nos resíduos e é estimada por Máxima Verossimilhança). Os modelos são especificados, de acordo com Kopczewska (2021), da seguinte forma:

$$
\begin{aligned}
& \frac{1}{T} \ln \left(\frac{y_{i, t+1}}{y_{i, 0}}\right)=\beta_{1}+\beta_{2} \ln \ln \left(Y_{i, o}\right)+\mu_{i}, \text { onde } \mu_{i}=\lambda W \epsilon_{i}+u \\
& \frac{1}{T} \ln \left(\frac{y_{i, t+1}}{y_{i, 0}}\right)=\beta_{1}+\beta_{2} \ln \ln \left(Y_{i, o}\right)+\rho W \frac{1}{T} \ln \left(\frac{y_{i, t+1}}{y_{i, 0}}\right)+\mu_{i} \\
& \frac{1}{T} \ln \left(\frac{y_{i, t+1}}{y_{i, 0}}\right)=\beta_{1}+\beta_{2} \ln \ln \left(Y_{i, o}\right)+\lambda W \frac{1}{T} \ln \left(\frac{y_{i, t+1}}{y_{i, 0}}\right)+\mu_{i}, \quad \text { onde } \mu_{i}=\rho W \epsilon_{i}+u
\end{aligned}
$$

Em que:

$W$ = matriz de pesos espaciais que captura as relações de vizinhança;

$\lambda=$ coeficiente auto regressivo de erro espacial;

$u$ = resíduo do erro espacial;

$\rho=$ coeficiente de defasagem espacial;

40 Diagrama de Dispersão / de Moran é distribuído em quadrantes que representam quatro tipos de associação linear espacial, sendo que o quadrante Alto-Alto-AA representa agrupamento de cidades com elevados valores da variável de interesse e cercadas por vizinhos com altos valores também. O quadrante Baixo-Alto-BA considera a variável e interesse com baixo valor e com vizinhos com altos valores. O quadrante Baixo-Baixo -BB refere-se a um grupo de municípios com baixo valor para variável de interesse rodeado por cidades com valores também baixos. $O$ quadrante Alto-Baixo $-A B$ diz respeito à região com variável de interesse com alto valor e com regiões vizinhas com valores baixos (ALMEIDA, 2012).

5 Modelo desenvolvido por LEE; LIU (2006). 
Em conformidade com Casagrande et al. (2013), pode-se notar que os modelos apresentados nas equações 5-8, através da adição do termo $\sum_{(i=1)}^{k} \beta_{k} X_{i, t}$ em cada uma das equações, tornam-se a especificação da $\beta$-convergência condicional.

\subsection{Fonte de dados}

As informações dos municípios do Rio Grande do Norte são do Anuário Estatístico do Instituto de Desenvolvimento Sustentável e Meio Ambiente do Rio Grande do Norte (IDEMA), Instituto Brasileiro de Geografia e Estatística (IBGE) e do Programa das Nações Unidas para o Desenvolvimento (PNUD) para os anos de 1991 e 2014. Seguindo a literatura de convergência, utilizou-se a taxa de crescimento da renda per capita dos municípios para testar a hipótese de convergência absoluta e condicional e sigma convergência.

Desta forma, a base de dados compreende 167 observações. Na análise exploratória espacial e nas estimações dos modelos de econometria espacial, assim como todos os testes, foi utilizado o software GeoDa e Stata versão 16.0, respectivamente.

Foram utilizadas como variáveis explicativas o logaritmo da renda per capita no início do período (Inrendapc91) para o modelo de convergência absoluta e as variáveis anos médios de estudo (e_anosestudo) e esperança de vida ao nascer (Espvida) nos modelos de convergência condicional.

De acordo com a literatura de crescimento econômico, como Mankiw, Romer e Weil (1995), Romer (1991) e no caso brasileiro, entre outros, Vieira (2009) e Justo (2010), consideramos o nível de educação como proxy para capital humano. Neste caso, é esperada uma correlação positiva desta variável com a taxa de crescimento dos municípios. Conforme a Nova Geografia Econômica (NGE), foi incluída entre os determinantes a esperança de vida ao nascer para captar condições de saúde da população.

\section{Resultados}

Conforme Barro e Sala-i-Martin (1992) uma relação negativa entre a renda per capita inicial e a taxa média de crescimento do PIB per capita é uma evidência da ocorrência de convergência. Portanto, na presente seção, são apresentados os resultados e discussões acerca da estatística descritiva, $\beta$-convergência absoluta e condicional, $\sigma$-convergência e da dependência espacial. 


\subsection{Estatística descritiva}

A Tabela 2 traz a estatística descritiva das variáveis utilizadas nas estimações dos modelos de convergência da renda per capita entre os municípios do Rio Grande do Norte. Observa-se que há municípios que apresentaram crescimento de cerca de $2 \%$ até outros que cresceram cerca de $8 \%$.

TABELA 2 - ESTATÍSTICA DESCRITIVA DAS VARIÁVEIS

\begin{tabular}{ccccc}
\hline Variável & Média & Desvio padrão & Mínimo & Máximo \\
\hline txrendapc & 0.049584 & 0.011273 & 0.024383 & 0.078983 \\
Lnrenda91 & 4.748887 & 0.333544 & 4.041998 & 6.206878 \\
E_ANOSESTUDO & 6.693413 & 1.003506 & 2.19 & 8.89 \\
Espvida & 58.79904 & 3.006968 & 53.88 & 66.59 \\
\hline
\end{tabular}

FONTE: Contruída com base no IDEMA (2014); IBGE (2020); PNUD (2020).

Também se observa variabilidade entre as demais variáveis e que, possivelmente, terão impacto nos resultados dos modelos estimados.

\subsection{Análise da $\beta$-convergência absoluta}

Uma vez rejeitada a distribuição espacial aleatória dos dados, indicando a necessidade de incorporar o efeito de vizinhança, parte-se para a estimação dos modelos que apreendem a convergência absoluta da renda per capita entre os municípios norterio-grandenses.

A Tabela 4 traz a estimação dos modelos das equações (1), (5), (6) e (7). Em todos os modelos, dada a significância do coeficiente da variável explicativa, observa-se a convergência absoluta da renda per capita entre os municípios do RN para o período 19912014, apontando, assim, a robustez desse resultado. Ou seja, os municípios com menor renda per capita tendem a crescer mais rapidamente que os municípios que têm maior renda per capita. O modelo (1) é apenas para efeito de comparação e para realização dos testes de dependência espacial, haja vista que os testes iniciais apontaram para a necessidade de incorporar os efeitos espaciais. A estimação dos demais modelos é para selecionar o que melhor representa a dinâmica da convergência. 
TABELA 4 - TESTES DE $\beta$ CONVERGÊNCIA ABSOLUTA COM QUATRO MODELOS: REGRESSÃO LINEAR SIMPLES (1); MODELO DE ERRO ESPACIAL (2); MODELO DE DEFASAGEM ESPACIAL (3); MODELO ML SARAR (4)

\begin{tabular}{|c|c|c|c|c|}
\hline & $\begin{array}{c}\text { txrendapc } \\
\text { (1) }\end{array}$ & $\begin{array}{c}\text { txrendapc } \\
\text { (2) }\end{array}$ & $\begin{array}{c}\text { txrendapc } \\
\text { (3) }\end{array}$ & $\begin{array}{c}\text { txrendapc } \\
\text { (4) }\end{array}$ \\
\hline \multicolumn{5}{|l|}{ main } \\
\hline \multirow[t]{2}{*}{ Inrenda91 } & $-0.0219^{* * *}$ & $-0.0238^{* * *}$ & $-0.0215^{* * *}$ & $-0.0221^{* * *}$ \\
\hline & $(-8.29)$ & $(-10.67)$ & $(-10.54)$ & $(-11.08)$ \\
\hline \multirow[t]{2}{*}{ _cons } & $0.154^{* * *}$ & $0.163^{* * *}$ & $0.149^{* * *}$ & $0.155^{* * *}$ \\
\hline & -12.31 & -15.28 & -13.42 & -16.23 \\
\hline \multicolumn{5}{|l|}{ lambda } \\
\hline \multirow[t]{2}{*}{ _cons } & & $0.243^{* *}$ & & -0.105 \\
\hline & & -2.97 & & $(-0.82)$ \\
\hline \multicolumn{5}{|l|}{ sigma } \\
\hline \multirow[t]{2}{*}{ _cons } & & $0.00825^{* * *}$ & $0.00853^{* * *}$ & \\
\hline & & $(-18.1)$ & $(-18.27)$ & \\
\hline \multicolumn{5}{|l|}{ rho } \\
\hline \multirow[t]{2}{*}{ _cons } & & & 0.0575 & \\
\hline & & & $(-0.83)$ & \\
\hline \multicolumn{5}{|l|}{ sigma2 } \\
\hline \multirow[t]{2}{*}{ _cons } & & & & $0.0000728^{* * *}$ \\
\hline & & & & $(-9.18)$ \\
\hline Número de Observações & 167 & 167 & 167 & 167 \\
\hline LM & & $7.472(0.006)$ & $0.701(0.403)$ & \\
\hline Teste de Robustez LM & & $13.878(0.000)$ & $7.106(0.008)$ & \\
\hline $\mathrm{R}^{2}$ & 0.4214 & 0.4318 & 0.4089 & 0.417 \\
\hline $\mathrm{F}$ & $68.71^{* * *}$ & $118.61^{* * *}$ & $90.39^{* * *}$ & $92.14^{* * *}$ \\
\hline Velocidade de convergência & 0.0258 & 0.0285 & 0.0252 & 0.0261 \\
\hline Meia vida & 31.3 & 28.78 & 31.89 & 31.02 \\
\hline
\end{tabular}

FONTE: Estimações com base nos dados do IBGE (2020); PNUD (2020); IDEMA (2014).

NOTA: $t$ calculado entre parêntesis. ${ }^{*} p<0.05,{ }^{* *} p<0.01,{ }^{* * *} p<0.001$.

A robustez dos resultados dos testes aponta que o modelo de erro espacial é o mais adequado. O coeficiente da variável da renda per capita no período inicial é de -0,0238. O coeficiente da variável que apreende a autocorrelação espacial também é significante e positivo com valor de 0,243 . Isso significa que choques apresentam autocorrelação positiva, ou seja, altos valores desses efeitos não modelados ocasionam choques de altos valores nos vizinhos, bem como choques de baixos valores geram baixos valores de choques nos vizinhos. Dito de outra forma, choques na taxa de crescimento de municípios com alta taxa de crescimento afetará fortemente os vizinhos, já choques na taxa de crescimento de municípios com baixa taxa de crescimento gera baixo efeito nos vizinhos. 
A velocidade de convergência é de 0.0285 e a meia vida é de 28.78 anos. Ou seja, os municípios do Rio Grande do Norte levariam quase trinta anos para atingir a metade da renda per capita no estado estacionário. Resultados nessa direção também foram encontrados por Reis, Araújo e Lima (2018) para a Região Nordeste.

\subsection{Análise da $\beta$ convergência condicional}

Na sequência, a Tabela 5 apresenta os resultados da equação (2), (5), (6) e (7) e (8), ou seja, da convergência condicional de renda per capita. A especificação da Equação 2 visa a diminuir o viés de variável omitida presente na Equação 1, com a introdução de variáveis associadas ao capital humano de cada município, controlando assim, diferenças relevantes no período inicial ${ }^{6}$.

Em todos os modelos os coeficientes das variáveis explicativas são significantes. Uma regularidade observada é a de que todos os modelos apontam para a convergência condicional. Ou seja, os municípios com menor renda per capita crescem mais rapidamente que aqueles que apresentam maior renda per capita, mas cada um cresce para o seu estado estacionário de longo prazo.

6 Os erros padrão são robustos à heterocedasticidade. O teste de Fator de Inflação de Variância (FIV) não apontou problema de multicolinearidade. 
TABELA 5 - TESTE DE $\beta$-CONVERGÊNCIA CONDICIONAL DE RENDA PER CAPITA PARA OS MUNICÍPIOS DO RN DE 1991 A 2014

\begin{tabular}{|c|c|c|c|c|c|}
\hline & $\begin{array}{c}\text { (1) } \\
\text { txrendapc }\end{array}$ & $\begin{array}{c}(2) \\
\text { txrendapc }\end{array}$ & $\begin{array}{c}\text { (3) } \\
\text { txrendapc }\end{array}$ & $\begin{array}{c}\text { (4) } \\
\text { txrendapc }\end{array}$ & $\begin{array}{c}\text { (5) } \\
\text { txrendapc }\end{array}$ \\
\hline \multicolumn{6}{|l|}{ Txrendapc } \\
\hline \multirow[t]{2}{*}{ Inrenda91 } & $-0.0280^{* * *}$ & $-0.0320^{* * *}$ & $-0.0274^{* * *}$ & $-0.0279 * * *$ & $-0.0280^{* * *}$ \\
\hline & $(-12.00)$ & $(-12.71)$ & $(-11.74)$ & $(-10.63)$ & $(-12.00)$ \\
\hline \multirow[t]{2}{*}{ E_anosestudo } & $0.00181^{* *}$ & $0.00210^{* *}$ & $0.00180^{* *}$ & $0.00181^{*}$ & $0.00181^{* *}$ \\
\hline & -2.66 & -3.27 & -2.67 & -2.55 & -2.66 \\
\hline \multirow[t]{2}{*}{ Espvida } & $0.000778^{* *}$ & $0.00108^{* * *}$ & $0.000821^{* *}$ & $0.000777^{* *}$ & $0.000778^{* *}$ \\
\hline & -2.98 & -4.15 & -3.15 & -2.9 & -2.98 \\
\hline \multirow[t]{2}{*}{ _cons } & $0.125^{* * *}$ & $0.124^{* * *}$ & $0.115^{* * *}$ & $0.125^{* * *}$ & $0.125^{* * *}$ \\
\hline & -9.96 & -9.52 & -8.16 & -8.17 & -9.96 \\
\hline \multirow[t]{3}{*}{ Lambda } & -0.109 & & & & \\
\hline & & $0.349^{* * *}$ & & $-0.110^{* *}$ & -0.109 \\
\hline & $(-0.82)$ & -4.65 & & $(-2.86)$ & $(-0.82)$ \\
\hline \multicolumn{6}{|l|}{ Rho } \\
\hline \multirow[t]{2}{*}{ _cons } & 0.0646 & & 0.0908 & & 0.0646 \\
\hline & -0.09 & & -1.37 & & -0.09 \\
\hline \multicolumn{6}{|l|}{ sigma2 } \\
\hline \multirow[t]{2}{*}{ _cons } & $0.0000648^{* * *}$ & & & & $0.0000648^{* * *}$ \\
\hline & -9.15 & & & & -9.15 \\
\hline \multicolumn{6}{|l|}{ Sigma } \\
\hline \multirow[t]{2}{*}{ _cons } & & $0.00747^{* * *}$ & $0.00801^{* * *}$ & & \\
\hline & & -17.92 & -18.26 & & \\
\hline $\mathrm{N}$ & 167 & 167 & 167 & 167 & 167 \\
\hline $\mathrm{R} 2$ & & 0.4733 & 0.4271 & 0.4671 & 0.45 \\
\hline $\mathrm{F}$ & & $49.00^{* * *}$ & $25.13^{* * *}$ & $123.04^{* * *}$ & $158.02^{* * *}$ \\
\hline LM & & $15.26^{* * *}$ & 1.77 & & \\
\hline Robust LM & & $21.58^{* * *}$ & $8.09 * * *$ & & \\
\hline Velocidade de convergência & 0.0348 & 0.0414 & 0.0339 & 0.0348 & 0.0348 \\
\hline Meia Vida & 24.41 & 21.31 & 24.95 & 24.41 & 24.41 \\
\hline
\end{tabular}

FONTE: Elaborado com base nos dados do IBGE (2020); PNUD (2020); IDEMA (2014).

Nota: $t$ calculado entre parêntesis [1]. ${ }^{*} \mathrm{p}<0.05,{ }^{* *} \mathrm{p}<0.01,{ }^{* * *} \mathrm{p}<0.001$.

De modo geral, a inclusão de controles associados ao capital humano acelera o processo de centralização de renda entre os municípios. A escolaridade mais elevada e maior esperança de vida aumentam o crescimento da renda per capita dos municípios analisados. Da mesma forma que na estimação da convergência absoluta também foram estimados vários modelos, incluindo o modelo MQO, para servir de parâmetro e para a realização 
dos testes de dependência espacial. Os testes apontaram a necessidade de incorporação do efeito espacial e o modelo mais apropriado foi o modelo (2), ou seja, o modelo de erro espacial. Assim, tem-se que no modelo de convergência condicional a convergência da renda per capita ocorre de forma mais acelerada com a velocidade de 0,0414 e há uma redução na meia vida para cerca de 21 anos.

Oajustedomodelo da convergência condicional tambémésuperior.Aautocorrelação espacial é de 0,349. Também é superior ao resultado do modelo de convergência absoluta. Esse valor também indica que choques não modelados na taxa de crescimento da renda per capita fazem com que o efeito nos municípios com alta taxa influenciem fortemente os vizinhos, bem como municípios com baixa taxa de crescimento influenciem pouco os seus vizinhos. Esses resultados estão em acordo com a literatura que aborda esse tema para o Brasil e para outros estados brasileiros a exemplo dos resultados encontrados por Justo (2021), Silveira, Silva e Carvalho (2010), Lima (2015) e Casagrande, Hoeckel e Santos (2016).

\subsection{Análise da Sigma $\sigma$-convergência}

A convergência $\sigma$ se manifesta através da diminuição da dispersão da renda per capita entre os diferentes municípios ao longo do tempo, ou seja, ocorre se as variabilidades das economias diminuem com o passar do tempo, ratificando assim a hipótese de $\beta$-convergência. Significa, ainda, que as assimetrias entre os municípios estão atenuando-se progressivamente. O teste $\sigma$ pode ser apurado através do desvio-padrão ou do coeficiente de variação, onde um resultado igual a zero corresponde à convergência perfeita (SILVA; RIBEIRO, 2013).

Na Tabela 6 são apresentados os valores do coeficiente de variação entre os anos de 2000 e $2014^{7}$ para o teste da $\sigma$-convergência. Vale ressaltar que se houver um decaimento dos coeficientes de variação no decorrer dos anos, haverá evidências da ocorrência de $\sigma$-convergência entre os municípios norte-rio-grandenses, mostrando que a discrepância entre eles diminuiu no decorrer dos anos, confirmando assim a hipótese de $\beta$-convergência.

7 A análise do sigma convergência para este recorte temporal se deu em virtude da disponibilidade de dados. 
TABELA 6 - COEFICIENTES DE VARIAÇÃO DA RENDA PER CAPITA DOS MUNICÍPIOS DO RIO GRANDE DO NORTE ENTRE OS ANOS DE 2000-2014

\begin{tabular}{|c|c|c|c|c|c|c|c|c|c|c|c|c|c|c|c|}
\hline ANO & 000 & 001 & 2002 & 003 & 2004 & 2005 & 2006 & 2007 & 2008 & 2009 & 2010 & 2011 & 2012 & 2013 & 014 \\
\hline $\mathrm{C}$ & 1 & 0,4 & 0,56 & 0,42 & 0,43 & 0,47 & 0,48 & 0,44 & 0,46 & 0,39 & 0,47 & 0,46 & 0 & 0,51 & 0,46 \\
\hline
\end{tabular}

Fonte: Estimações com base nos dados do IBGE (2020); PNUD (2020); IDEMA (2014).

Embora se observe que não ocorreu uma queda linear da dispersão do PIB per capita entre os municípios norte-rio-grandenses no período analisado, a dispersão ocorre entre os anos inicial e final, indicando a ocorrência da concentração de renda.

\subsection{Análise Exploratória Espacial}

Diante da ampla variedade de matrizes de pesos espaciais disponíveis, uma missão do pesquisador é encontrar a matriz de ponderação espacial adequada. O ideal, segundo Almeida (2012), é que essa matriz possa capturar toda autocorrelação espacial subjacente. Assim, foi estimada uma regressão linear por Mínimos Quadrados Ordinário (MQO), usando o índice I de Moran para um conjunto de matrizes W. Foi selecionada a matriz de pesos que gerou o maior valor da estatística I de Moran estatisticamente significante. Dessa forma, a matriz selecionada foi a matriz k-Nearest (com dois vizinhos) ${ }^{8}$.

Segundo Almeida (2012), o primeiro passo da Análise Exploratória de Dados Espacial (AEDE) é para verificar a hipótese de que os dados são distribuídos aleatoriamente no espaço. A Tabela 3 traz o resultado do teste I de Moran. Assim, rejeita-se a hipótese de aleatoriedade espacial dos dados.

TABELA 3 - RESULTADOS DO ÍNDICE I DE MORAN PARA A TAXA DE CRESCIMENTO DA RENDA PER CAPITA DO RN NO PERÍODO 1991-2014

\begin{tabular}{cccccc}
\hline \multicolumn{7}{c}{ Índice de Moran I } \\
\hline Variáveis & $\mathrm{I}$ & $\mathrm{E}(\mathrm{I})$ & Desvio padrão $(\mathrm{I})$ & $\mathrm{Z}$ & p-valor $^{*}$ \\
\hline txrendapc & 0.177 & -0.006 & 0.071 & 2.577 & 0.005 \\
\hline
\end{tabular}

FONTE: Estimações com base nos dados do IBGE (2020); PNUD (2020); IDEMA (2014).

8 Foram testados os seguintes tipos de Matrizes: rainha, distância inversa, k-vizinhos (2, 3, 4 e 5). 
Uma outra alternativa também utilizada foi a análise do gráfico I de Moran. 0 diagrama de dispersão foi construído com a variável taxa de crescimento da renda per capita municipal. O resultado pode ser visto na Figura 1.

\section{FIGURA 1 - GRÁFICO IDE MORAN PARA TAXA DE CRESCIMENTO DA RENDA PER CAPITA O RN NO PERÍODO 1991-2014}

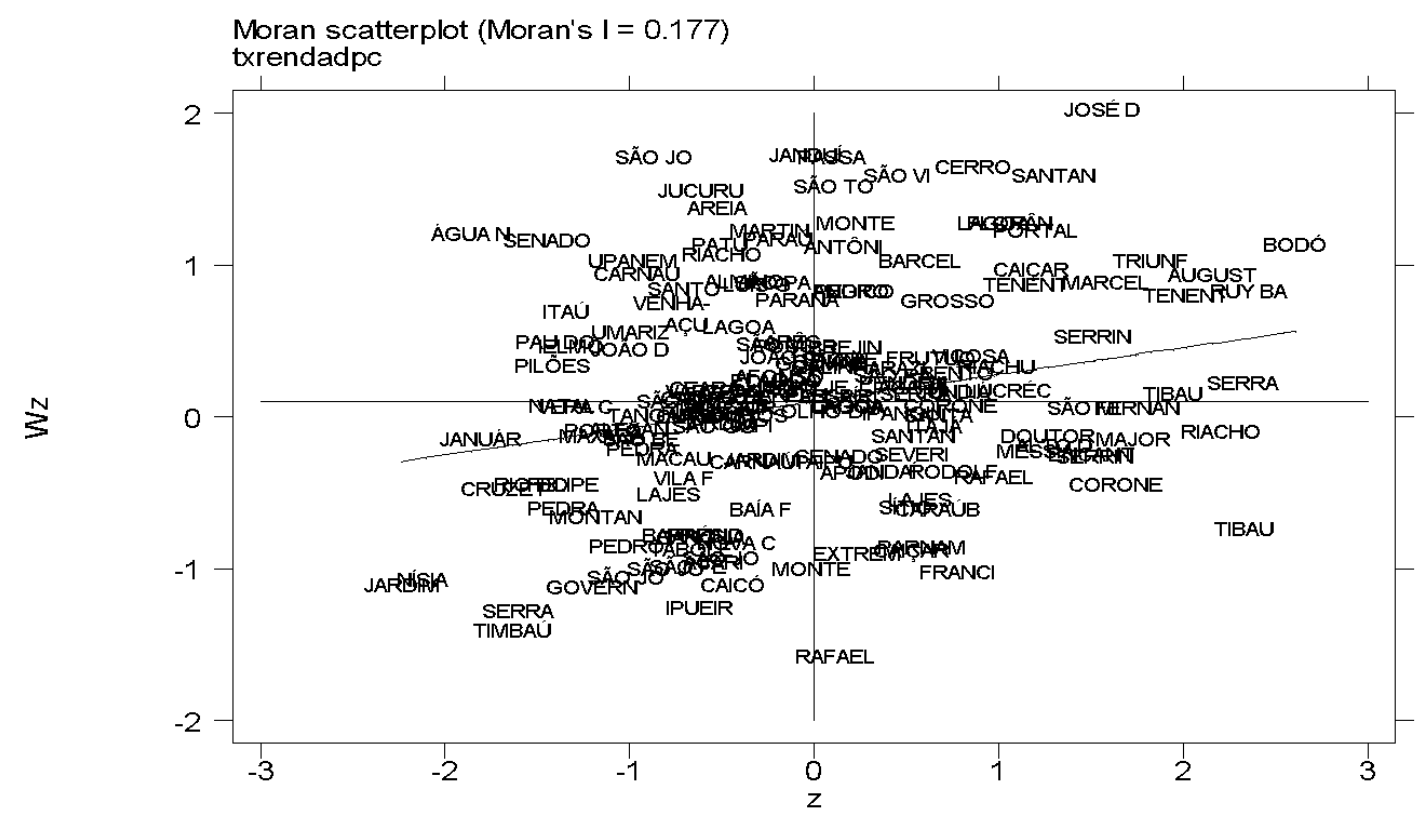

FONTE: Elborado com base nos dados do IBGE (2020); PNUD (2020); IDEMA (2014).

A Figura 2 mostra pontos de concentração de municípios com distintos padrões de crescimento. Há clusters nos quatro quadrantes: Alto-Alto (São Fernando, Jardim de Piranhas, Timbaúba dos Batistas, Serra Negra do Norte, São João do Sabugi e Ipueira), AltoBaixo (Natal, Nisia Floresta, São João do Mipibu e Ares), Baixo-Baixo (São Fernando, Jardim de Piranhas, Timbaúba dos Batistas, Serra Negra do Norte, São João do Sabugi e Ipueira) e Baixo-Alto (Baraúnas, Tibau, Areia Branca, Assu, Grosso, Serra do Mel e Mossoró) 


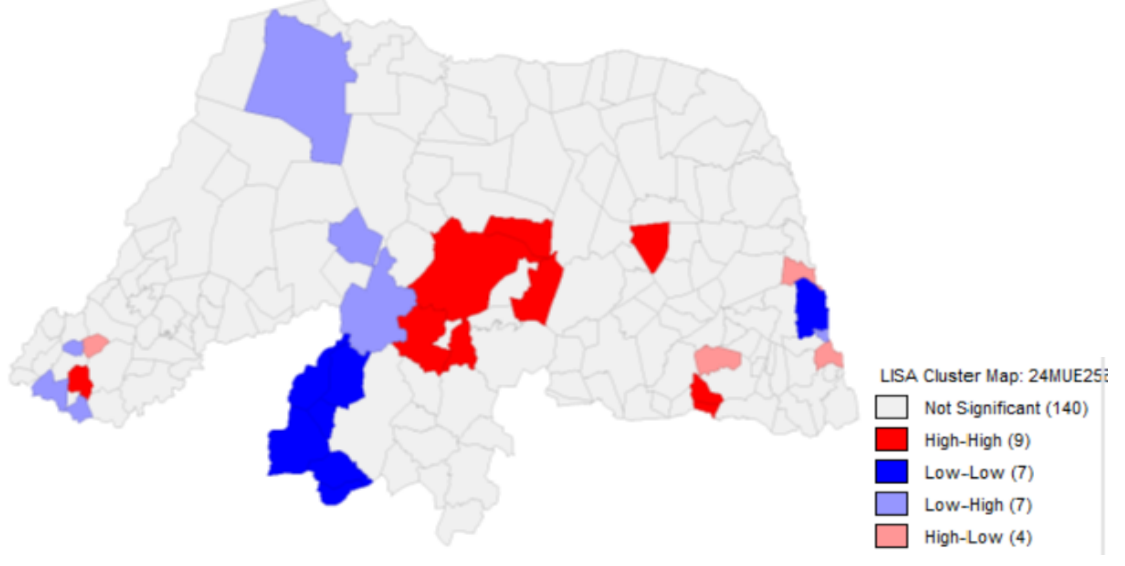

FONTE: Elaborado com base nos dados do IBGE (2020); PNUD(2020); IDEMA (2014).

A presença de clusters espaciais da dinâmica de crescimento é confirmada pelos resultados obtidos usando a metodologia LISA (Local Indicators of Spatial Assocciation). Esta metodologia permite fazer uma análise local do padrão espacial apresentada pelos dados levando em consideração a influência espacial em determinadas regiões ao passo que em outras regiões estes agrupamentos não são estatisticamente significantes (VIEIRA, 2009).

A Figura 3 apresenta a distribuição espacial da taxa de crescimento do produto entre 1991 e 2014. Aqui, também se observa que apesar da renda per capita ser baixa ainda assim ela não é distribuída uniformemente entre os municípios norte-rio-grandenses. Observase que há uma distribuição da taxa de crescimento da renda per capita dos municípios de forma quase equitativa entre os quintis, ou seja, em torno de trinta e três municípios por cada quintil da distribuição da renda. Contudo, a renda per capita média no último quintil é duas vezes maior em relação ao primeiro. 
FIGURA 3 - TAXA DE CRESCIMENTO DA RENDA PER CAPITA DOS MUNICÍPIOS NORTE-RIOGRANDENSES: 1991- 2014

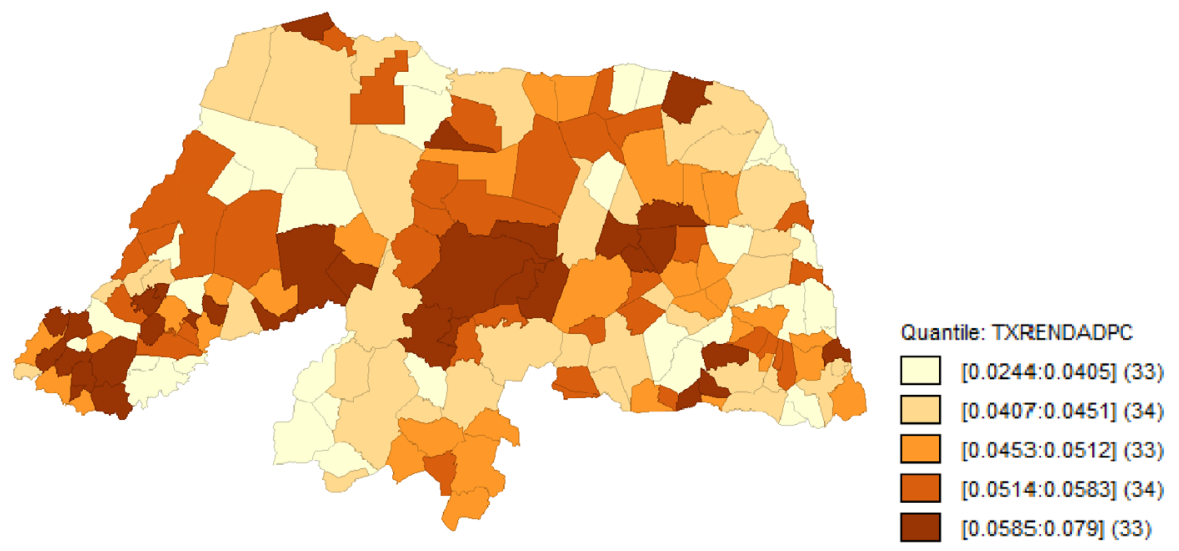

FONTE: Elaborado com base nos dados do IBGE (2020); PNUD (2020); IDEMA (2014).

\section{Conclusões}

O presente trabalho buscou uma melhor compreensão do processo de crescimento econômico dos municípios do Rio Grande do Norte para o período de 1991 a 2014. O trabalho investigou a convergência absoluta, condicional e a convergência sigma desse crescimento incorporando os efeitos de vizinhança.

Os resultados apontaram a necessidade de se incorporar a dependência espacial nos modelos estimados para corrigir o viés nos estimadores dos modelos de convergência. A hipótese de concentração de renda foi aceita nas três categorias testadas. A elevação da escolaridade e da esperança de vida colaborou para elevar o crescimento dos municípios do Rio Grande do Norte. O modelo de erro espacial mostrou-se mais apropriado. No modelo de convergência condicional, a velocidade de convergência é mais elevada e a meia vida se reduz. A velocidade de convergência é de 4,14\% e a meia vida de cerca de 21 anos.

Assim, pode-se observar que apesar das desigualdades econômicas existentes no estado ainda serem acentuadas, houve nos últimos anos uma redução deste diferencial. Apesar de que a convergência ocorre de forma muito lenta, ainda que em padrões mais elevados que os encontrados para outros estados brasileiros apontados na literatura. Dessa forma, há espaço para adoção de políticas que acelerem a convergência da renda per capita como a elevação da escolaridade formal, ações que reduzam o abandono e a repetência e políticas públicas na área de saúde que elevem a esperança de vida, como por exemplo, políticas de saneamento e prevenção de doenças cardiovasculares apontadas na 
literatura como de forte impacto no aumento da esperança de vida, haja vista que foram estas as variáveis que foram apontadas nos resultados com maior impacto para acelerar a convergência de renda entre os municípios, reduzindo o tempo necessário para que a renda de cada município atinja seu estado estacionário.

Este trabalho avança na literatura ao preencher uma lacuna ao apresentar as trajetórias de crescimento da renda per capita para o Rio Grande do Norte com os dados mais recentes disponíveis para esta economia e indicando a necessidade de incorporar os efeitos espaciais nos modelos que testam a convergência de renda para o estado. Outra contribuição é a identificação que a velocidade de convergência de renda no estado é mais elevada comparativamente à Região Nordeste como um todo, o que sugere que as economias dos estados menos pujantes da região podem ter suas peculiaridades econômicas e sociais que os distingue dos estados com maior participação na economia regional, a exemplo da Bahia, Pernambuco e Ceará.

Contribuições futuras a esse estudo vão no sentido de obtenção de dados de outras variáveis e um período mais amplo.

\section{Referências}

ABITANTE, K. G. Desigualdade no Brasil: um estudo sobre convergência de renda. Pesquisa \& Debate. Revista do Programa de Estudos Pós-Graduados em Economia Política, v. 18, n. 2 (32), 2007.

ALMEIDA, E. Econometria especial aplicada. Campinas: Alínea, 2012.

ANSELIN, L.; BERA, A.; FLORAX, R. J. G. M.; YOON, M. Simple diagnostic tests for spatial dependence. Reg. Sci. Urban Econ., v. 26, p. 77-104, 1996.

BAUMOL, William J. Productivity growth, convergence, and welfare: what the long-run data show. The american economic review, p. 1072-1085, 1986.

BARRO, R. J., SALA-i-MARTIN, X. Economic growth. 1a edição. Massachusetts: MIT Press, 1995. 539 p.

CASAGRANDE, Dieison Lenon; OLIVEIRA HOECKEL, Paulo Henrique; SANTOS, Cezar Augusto Pereira dos. Convergência de renda no Rio Grande do Sul: uma análise de 2001 a 2013. In: ENCONTRO DE ECONOMIA GAÚCHA, 8., 2016, Porto Alegre.

COELHO, R. L.; FIGUEIREDO, L. Uma análise da hipótese da convergência para os municípios brasileiros. Revista Brasileira de Economia, v. 61, n. 3, p. 331-352, 2007.

CHAGAS, André Luís Squarize. TONETO JUNIOR, Rudinei. Fatores determinantes do crescimento local: evidência a partir de dados dos municípios brasileiros para o período 1980-1991. Pesquisa e Planejamento EconômicoPPE, v. 33, n. 2, 2003.

CLINCH, J. Peter; O'NEILL, Eoin. Applying spatial economics to national spatial planning. Regional Studies, v. 43, n. 2, p. 157-178, 2009. 
CRAVO, T., SOUKIAZIS, E. Human capital as a conditioning factor to the convergence process among the Brazilian States. CEUNEUROP, Working Paper n 35, February,2006.

CRUZ, Italo Spinelli da et al. Dependência espacial e convergência local da renda entre os municípios do Nordeste: 2000-2010. 83f. Dissertação de mestrado apresentado à faculdade de Ciências Sociais Aplicadas, Programa de Pós-Graduação em Economia, Universidade Federal de Sergipe. Sergipe, 2013.

DE OLIVEIRA DAVID, Geovani Aparecido; MEYER, Leandro Garcia; DA PAIXÃO, Michel Augusto Santana. Convergência de renda nos municípios do estado do Paraná: uma análise empírica no período 20022015. Economia e Desenvolvimento, v. 31, p. 12, 2019.

DRUKKER, D. M.; PRUCHA, I. R.; RACIBORSKIR. Maximum-likelihood and generalized spatial two-stage leastsquares estimators for a spatial-autoregressive model with spatial-autoregressive disturbances. Working paper, University of Maryland, Department of Economics, 2011.

ERTUR, C.; KOCH, W. Growth, technological interdependence and spatial externalities: theory and evidence. Journal of Applied Econometrics, v. 22. n. 6, p. 1033-1062, 2007.

FERREIRA, P.C; ELLERY JÚNIOR, R.G. Convergência entre a renda per capita dos estados brasileiros. Revista de Econometria, Rio de Janeiro, v. 16, n. 1, p. 83-103, 1996.

FLORAX, R. J. G. M.; FOLMER, H.; REY, S. J. Specification searches in spatial econometrics: the relevance of Hendry's methodology. Regional Science and Urban Economics, v. 33, n. 5, p. 557-579, 2003.

GOMES, Rafaela Rodrigues et al. Convergência de renda: uma análise para os estados nordestinos no período de 1995-2009. 97f. Dissertação (Mestrado Profissional em Desenvolvimento Regional e Gestão de Empreendimentos Locais) - Universidade Federal do Sergipe, São Cristóvão, 2013.

INSTITUTO BRASILEIRO DE GEOGRAFIA E ESTATÍSTICA (IBGE). Disponível em: https://www.ibge.gov.br/portalnossos-sites/nossos-sites.html. Acesso em: 28 mar. 2020.

INSTITUTO DO DESENVOLVIMENTO SUSTENTÁVEL E MEIO AMBIENTE DO RIO GRANDE DO NORTE (IDEMA). Produto Interno Bruto do Estado e dos Municípios 2010-2014. Disponível em: http://adcon.rn.gov.br/ ACERVO/idema/DOC/DOC000000000138927.PDF. Acesso em: 28 mar. 2020.

JUSTO, W. R.Crescimento econômico e convergência de renda damesorregião do Araripe: uma abordagem espacial. Revista economia emdebate (URCA), v. 1, p. 01-20, 2011.

JUSTO, Wellington Ribeiro. Crescimento Econômico dos Municípios Baianos de 2000 a 2010 à luz da Nova Geografia Econômica e da Econometria Espacial. Revista Análise Econômica e Políticas Públicas-RAEPP, v. 1, n. $01,2021$.

KOPCZEWSKA, K. Applied spatial statistics and econometrics: data analysis in R. , New York: Routledge, 2021.

LEE, L. F.; XIAODONG, Liu. Efficient GMM estimation of a spatial autoregressive model with autoregressive disturbances. Manuscript, Ohio State University, 2006.

LIMA, A. F. R.; MARQUES, D. M. F; ALVES, L. B.; CASTRO, M. C. Análise de convergência de renda dos municípios goianos entre 2001 e 2011. Conjuntura Econômica Goiana, n. 34, 2015. Disponível em: http://www.imb. go.gov.br/down/analise_de_convergencia_de_renda_dos_municipios_goianos_entre_2001_e_2011.pdf . Acesso em: 20 mar. 2020.

LIM, U. Regional income club convergence in US BEA economic areas: a spatial switching regression approach. The Annals of Regional Science, v. 56, n. 1, p. 273-294, 2016. 
LOPES, J. L. Avaliação do processo de convergência da produtividade da terra na agricultura brasileira no período de 1960 a 2001. 208 f. Tese (Doutorado em Economia Aplicada) - Escola Superior de Agricultura Luiz de Queiroz, Universidade de São Paulo, Piracicaba, 2004.

MATOS FILHO, José Coelho; SILVA, Almir Bittencourt da; CARVALHO, Tiago Nunes. A convergência da renda nas microrregiões da Região Nordeste do Brasil. Revista Economia e Desenvolvimento, v. 11, n. 2, 2012.

MINCER, J. Schooling, experience, and earning. National Bureaus of Economic Research. New York: Columbia University Press, 1974.

PROGRAMA DAS NÇÕES UNIDAS PARA O DESENVOLVIMENTO (PNUD). Atlas do desenvolvimento do Brasil. Disponível em: http://www.atlasbrasil.org.br/. Acesso em: 02 fev. 2020.

REIS, Lucas David Ribeiro; ARAÚJO, Rogério César Pereira de; LIMA, João Ricardo Ferreira de. Convergência da renda per capita no Nordeste. In: CONGRESSO DA SOCIEDADE BRASILEIRA DE ECONOMIA, ADMINISTRAÇÃO E SOCIOLOGIA RURAL DO REGIONAL NORDESTE, 2018, Juazeiro, BA.

REY, Sergio J.; MONTOURI, Brett D. US regional income convergence: a spatial econometric perspective. Regional studies, v. 33, n. 2, p. 143-156, 1999.

SANDRONI, Paulo. Dicionário de economia do século XXI. Rio de Janeiro: Record, 2005.

SANTOS, Ricardo Bruno Nascimento dos et al. Crescimento econômico e clubes de convergência nos municípios mineiros: uma análise com modelo threshold. Revista EconomiA, 2012.

SILVA, Ana Paula Nunes; RIBEIRO, Hilton Manoel Dias; BASTOS, Suzana Quinet de Andrade. Análise de convergência de desenvolvimento multidimensional para regiões intermediárias de Minas Gerais. Revista Brasileira de Estudos Regionais e Urbanos, v. 13, n. 4, p. 561-580, 2019.

SILVA JÚNIOR, D. Aglomeração e desenvolvimento: evidências para municípios brasileiros. 104 f. Dissertação (Mestrado em Economia) - Faculdade de Economia, Administração e Contabilidade, Universidade de São Paulo, São Paulo, 2007.

SILVEIRA-NETO, R.; AZZONI, C. R. Location and regional income disparity dynamics: the Brazilian case. Papers in Regional Science, v. 85, n. 4, p. 599-613, 2006.

SOLOW, R. M. A Contribution to the Theory of Economic Growth. Quarterly Journal of Economics, v. 70, p. 65-94, 1956.

STÜLP, V. J.; FOCHEZATTO, A. A evolução das disparidades regionais no Rio Grande do Sul: uma aplicação de matrizes de Markov. Nova Economia, v. 14, n. 1, p. 39-66, 2004.

SWAN, T. W. Economic growth and capital accumulation. Economic Record, v. 32, p. 334-361, 1956.

VIEIRA, R. S. Crescimento econômico no estado de São Paulo: uma análise espacial [online]. São Paulo: Editora UNESP; São Paulo: Cultura Acadêmica, 2009. 103 p.

WOOLDRIDGE, Jeffrey M. Econometric analysis of cross section and panel data. MIT press, 2010. 